\title{
La heterogeneidad de las políticas sociales en México
}

\author{
Jorge Enrique Bracamontes Grajeda•
}

http://dx.doi.org/10.32870/espiral.v25i73.6579.g6129

La obra que editan Oscar Alfonso Martínez, Enrique Valencia y Luis Ignacio Román presenta un panorama dispuesto para el análisis actualizado de la política social en nuestro país, ya que en los capítulos correspondientes a este segundo volumen se integran diferentes posturas teóricas, así como gran diversidad de metodologías de investigación.

El volumen está organizado en cuatro partes sustantivas: 1) una sobre la política social de cuidados, 2) otra sobre la política social en su perspectiva territorial, 3) dos estudios inherentes al acceso al agua como objeto de bienestar social y derecho humano, y 4) un par de estudios concretos sobre educación y su evaluación como puntos de política social pendientes aún en la agenda nacional. La estructura del volumen obedece a un orden deductivo de análisis desde las temáticas

Profesor-Investigador de la Facultad de Trabajo Social de la Universidad Juárez del Estado de Durango, México. ORCID: http://orcid.org/00000002-3566-9893 jbgrajeda@gmail.com planteadas, y esto contribuye sensiblemente en la propia lectura y revisión de los textos, tanto como acervo metodológico como de teorización profunda, atributos que recomiendan por sí mismos la consulta del libro.

Oscar Alfonso Martínez Martínez, Enrique Valencia Lomelí, y Luis Ignacio Román Morales (eds.) (2016). La heterogeneidad de las políticas sociales en México: instituciones, derechos sociales y territorio (vol. 2). México: Universidad Iberoamericana y Universidad de Guadalajara. 
En el capítulo 1, "La caída de la economía del cuidado", Osiris Marín presenta de forma sustancial cómo han sido impactadas las prácticas del cuidado en el hogar en Ciudad Juárez, Chihuahua. Dicho impacto está ligado en específico a las transformaciones del trabajo, a los cambios en la estructura familiar y a las dinámicas de convivencia y cuidados en la misma.

En el capítulo 2, Rocío Enríquez nos presenta en "La construcción social del cuidado: ¿individualización, familiarización o colectivización? Reflexiones a partir de debates contemporáneos" una discusión profunda acerca de las implicaciones que tiene la construcción social del cuidado como un eje rector de la política de cuidados, pese a que el Estado moderno sigue delimitando dicha práctica a la sola esfera privada. Dentro de esta discusión, incluso, quedan manifiestas las desigualdades de género que supone el sostenimiento del cuidado en las familias mexicanas.

Gabriela Pérez Yarahuán presenta el tercer capítulo en esta obra, que se titula "Situación actual y retos para la política social en materia de niñez y adolescencia en México. Análisis a partir de veintisiete programas federales". En dicho capítulo, se expone un análisis instrumental de la parte de la política social mexicana que tiene que ver con el mejoramiento de la calidad de vida del sector de la población de entre cero y diecisiete años. Entre los resultados más interesantes de esta propuesta analítica está, por ejemplo, que en la revisión hecha de los objetivos de los programas, los derechos sociales que atienden y la carencia o sistemas de carencia, no se destaca una correspondencia entre dichas dimensiones, es decir, lo que se juzga como alcance de los objetivos de programa no empata con la carencia que se intenta satisfacer o el derecho social que pretende cubrir, lo que demuestra la limitación del programa para resolver las necesidades sociales a cuya solvencia está destinado. 
"Transferencias monetarias para hogares con menores de edad de acuerdo con la ENIGH [Encuesta nacional de ingresos y gastos de los hogares] 2010: ¿focalizar o universalizar?" es, por su parte, un trabajo presentado por Luis Huesca, Anahely Medrano y Luis Gutiérrez Flores, en el que se aborda el estudio del potencial de las transferencias monetarias como estrategia para la reducción de la pobreza infantil. En concreto, se analiza la transferencia monetaria del programa Oportunidades, así como de apoyo alimentario, además de otras becas públicas y privadas. Como los programas van dirigidos a hogares con menores de edad, el supuesto es que dichas transferencias debería beneficiar de mejor forma a familias con dichos integrantes.

El capítulo se centra en que el beneficio de estos programas es marginal. En conclusión, las transferencias de Oportunidades son más regresivas en hogares sin menores de edad que en el caso contrario. En el caso de las becas, estas se han concentrado más en estratos medio y alto de ingresos. Estas conclusiones se desprenden del análisis de la función de progresividad de dichos ingresos sobre la satisfacción en las estructuras de necesidades en hogares con menores de edad y sin ellos.

En el quinto capítulo, "Cómo explicamos la movilidad social de los jóvenes beneficiarios de Oportunidades: contribución de algunas variables psicosociales”, propuesto por Joaquina Palomar Lever, se refresca la discusión sobre movilidad social a la luz de las bases argumentativas de los programas sociales como Oportunidades.

En dicho apartado, se apunta que los indicadores del programa Oportunidades son utilizados como un modo de medir la movilidad social. Los resultados son interesantes en lo general, sobre todo en aspectos como religiosidad y adhesión a grupos. En concreto, la movilidad positiva es propiciada por habilidades y competencias ligadas a la educación y limitadas ante las cuestiones psicológicas -la 
movilidad se presenta ante situaciones patológicas más que ante las que tendrían que ver con la llamada psicología positiva-. Estos resultados contradicen algunas afirmaciones presupuestas acerca del bienestar generado a partir de una mayor religiosidad o adhesión a grupos, ya que el opus de control interno o individual se subordina al de la religión o al de la pertenencia a grupos (opus de control externo).

Por su parte, Sandra Mancinas y Sagrario Garay exponen en "Envejecimiento y redes de apoyo informales en México" un análisis profundo de las redes de apoyo informales en que se promueve el sostenimiento y cuidado de la población envejecida. Se destaca en el texto el cambio constante en todos los aspectos de la red. Con la edad, no sólo van cambiando los tipos de apoyos que fluyen en ella, sino que disminuyen. Por ejemplo, el apoyo moral es uno de los tipos de apoyo más vulnerado, y dicha vulneración proviene de la familia o de las personas no familiares que pertenecen a la red de apoyo. Por lo anterior, es necesario profundizar en el estudio de las causas de la falta de reciprocidad, ya que la población adulta mayor proporciona apoyo a sus grupos no familiares y familiares, aunque proporcionalmente no parece haber correspondencia con el apoyo que recibe de esos mismos grupos.

"La alimentación como factor de bienestar: un análisis desde el programa Pensión Alimenticia para Adultos Mayores del Estado de México" es un estudio realizado por Gabriela Fuentes Reyes y Oscar Alfonso Martínez Martínez, en el que se trata de explicar cómo un apoyo social alimentario proporciona bienestar a la población adulta mayor. Se retoma sustancialmente la discusión sobre la definición de bienestar con el fin de proporcionar un marco de inteligibilidad al análisis del mismo en la población adulta mayor, análisis en que se destaca una caracterización general sobre lo tangible del bienestar - bienestar material u objetivo- y sobre sus características intrínsecas -denominadas usualmente 
como bienestar subjetivo-. Más que un artefacto generador de bienestar, el estudio alude a que la alimentación es tan sólo un complemento del mismo que beneficia la subsistencia. Los programas como los de apoyo alimentario no cubren las expectativas objetivas y discursivas que pretenden sus implementadores.

A su vez, en "Desigualdad intrarregional: las propensiones como indicio de desigualdad social crónica", Rosa María Ruvalcaba y Juan Carlos Ordaz presentan en detalle el conjunto de desigualdades sociales derivadas de una estructura de políticas públicas y sociales verticales, que discriminan entre territorios desarrollados y subdesarrollados, favorecidos y desfavorecidos, resaltando en estos últimos su carácter marginal, enunciado desde los autores como el México olvidado.

Las características territoriales diferentes, por ejemplo, entre norte/sur, territorio urbano/territorio rural, e inclusive territorio indígena/territorio no indígena, acentúan condiciones de marginación material en la gestión de recursos de infraestructura que necesariamente se vinculan con la reproducción de-también-vulnerabilidades sociales. Este capítulo destaca entonces tanto problemáticas históricamente no resueltas como problemáticas emergentes que vuelven todavía más compleja la gestión del territorio como ancla del desarrollo social.

"Satisfacción de beneficiarios de programas sociales como indicador de buen gobierno. El problema de identificar lo que demanda el ciudadano" es un estudio presentado por J. Mario Herrera Ramos, Alejandro Islas Camargo y Miriam Rodríguez Vargas, en el cual se describen con detalle las percepciones de la población respecto a su satisfacción con sus autoridades de Gobierno. En concreto, se destaca que no hay vinculación entre satisfacción de beneficiarios y calidad de Gobierno. Las medidas recomendadas de evaluación de la política social no son compatibles cuando se extrapolan los 
esquemas de evaluación del desempeño con este reflejado en la satisfacción sobre la relación ciudadano-Gobierno. Esto sugiere entonces la necesidad de cambios estructurales en la relación gobernabilidad-gobernanza, a fin de volver compatibles ambas dimensiones, la de la actuación gubernamental y la del bienestar ciudadano.

"Condiciones económicas y sociales de la marginación en el estado de Tamaulipas: una perspectiva desde cómo lo viven los actores" es un estudio presentado por Adolfo Rogelio Cogco Calderón, Jorge Alberto Pérez Cruz y Miriam Rodríguez Vargas, en el cual se exponen las consecuencias que en términos de marginalidad tiene para el estado de Tamaulipas la dispersión poblacional de las zonas rurales, así como la desventaja territorial que existe para acceder a la provisión de bienes y servicios públicos y la generación y distribución de la riqueza. Se enfatiza la estrategia de construir soluciones desde lo local en cada una de las comunidades en condiciones de marginación.

Rodrigo Flores Elizondo expone en "Derecho humano al agua y tarifas para el abasto urbano" una interesante reflexión en torno a cómo un buen manejo tarifario en los sistemas de agua potable puede proporcionar esquemas de bienestar y abatimiento de desigualdades sociales. Se describe como ideal un manejo tarifario del agua que por un lado sea congruente con los aspectos de la gestión del recurso agua, y por otro sea coherente con las necesidades sociales. Esta profunda reflexión pretende analizar, desde una perspectiva compleja, desde la presión sobre el recurso natural hasta el acceso al derecho humano al agua, pasando por las dificultades estructurales de las instituciones administradoras y distribuidoras del recurso.

El acceso al agua como objeto de política social se analiza en "Derechos y regímenes de rendición de cuentas. Otra mirada del agua potable y el saneamiento en la zona metropolitana de Xalapa, Veracruz", capítulo realizado por

\section{6}


Ernesto Isunza Vera. En este estudio, se integran analíticamente distintas dimensiones como la normatividad, las prerrogativas de Gobierno en distintos niveles, la gestión del recurso agua y su saneamiento, así como las formas en que debe gestionarse y los actores involucrados -o que debieran estar involucrados en dicha gestión-. En el contexto estudiado, existen rasgos del fenómeno singulares, como una gestión verticalista que no permite la llamada cogestión del agua potable y saneamiento, lo que vulnera estructuralmente el acceso al recurso o el ejercicio del derecho humano legalmente establecido por distintas instancias jurídicas. Esto señalaría la necesidad de formular estructuras que permitan, por ejemplo, acceder a esquemas de participación en gobernanza real.

Miguel Bazdresch Parada expresa en "La política educativa de evaluación de la educación y sus consecuencias sociales" una crítica objetiva de la política evaluativa del sistema educativo nacional. En el capítulo, destaca la reflexión sobre la incongruencia entre el proceso de evaluación educativa y su falta de repercusión en la gestión de la educación, que debieran estar encaminados a consolidar una educación de calidad que considerara las voces de todos los actores, es decir, familias, magisterio e instituciones. Aunque la evaluación es ya una práctica común en el sistema educativo, no refleja, como indica el autor, consecuencias sociales que justifiquen tanto el gasto como el esfuerzo en su realización.

Por último, la educación también es arena de discusión sobre la desigualdad, y en el capítulo "Los pueblos originarios en las políticas de educación intercultural: prácticas y desafíos del indigenismo en tiempos neoliberales", Bruno Baronnet destaca las condiciones estructurales que impiden la planificación autónoma que para su crecimiento requiere el subsistema de educación intercultural. En este sentido, el autor enfatiza la necesidad de dejar de experimentar la gestión de la educación bilingüe e indígena y a la vez consolidar 
su estructura centrada en lo microrregional, a fin de que las innovaciones pedagógicas sean precedente y continuum para la gestión del aprendizaje en los pueblos originarios de nuestro país. 